\title{
THE SAMPLE SIZE NEEDED FOR THE CALCULATION \\ OF A GLM TARIFF
}

BY

HANS SCHMITTER

\begin{abstract}
A simple upper bound for the variance of the frequency estimates in a multivariate tariff using class criteria is deduced. This upper bound is based exclusively on univariate statistics and can, therefore, be calculated before a GLM analysis is carried out. It can be used to estimate the number of claims that will be needed for a tariff calculation depending on the number of tariff criteria and the number of levels of each criterion.

The article is a revised version of a paper presented at the XXXIst ASTIN Colloquium in Porto Cervo.
\end{abstract}

\section{KEYWORDS}

Poisson, frequency, multiplicative tariff, generalised linear model, sample size.

\section{INTRODUCTION}

When the estimate of the Poisson parameter for identical risks is required to lie close to the true value (e.g. within $10 \%$ ) with high probability (e.g. 95\%), the number of observed claims must exceed a certain minimum which can be determined in a straightforward way. Let $\lambda$ be the Poisson parameter, $s$ the number of risks, $Y$ the Poisson-distributed number of claims and $y$ an observation of $Y$, i.e. the observed number of claims. This means

$$
\operatorname{Prob}\{|Y / s-\lambda| \leq c \lambda\} \geq p,
$$

when we write $c$ and $p$ instead of $10 \%$ and $95 \%$.

Using the normal approximation with expected value and variance equal to $\lambda s$ and rewriting (1) as

$\operatorname{Prob}\{|Y-\lambda s| / \sqrt{\lambda s} \leq c \sqrt{\lambda s}\} \geq p$

we have

$0.1 \sqrt{\lambda s} \geq 1.96$ 
in our example with $c=0.1$ and $p=0.95$ and hence $\lambda s \geq 384.16$. This means the expected number of claims must exceed 384.16. Estimating the expected $\lambda s$ by the observed number $y$ we thus get $y \geq 384.16$. Applying this result to the calculation of a tariff for identical risks one needs a sample with at least 385 claims (or any other minimum depending on appropriate values for $c$ and $p$ ) in order to determine the claims frequency with the precision required.

To the author's knowledge no such rules guaranteeing sufficient precision are known in the case of tariffs using several rating criteria. It is intuitively clear that the minimum sample size will increase as the number of criteria increases but whether or not the available data is extensive enough is not known in advance. Often only after time-consuming analyses does one discover that the statistical basis for the calculation of a sophisticated tariff was in fact too small.

The purpose of the present paper is to give simple rules for checking whether or not the available sample is large enough to allow the frequencies of a multivariate tariff to be calculated. The result is presented in the form of an upper bound which can be calculated based on simple statistics. If the sample is larger than this upper bound, then the frequencies can be determined with the required accuracy. If, on the other hand, the sample is smaller than the upper bound, then the pre-defined accuracy is not guaranteed.

\section{NOTATION}

We use the following notation:

$Y_{i} \quad$ Poisson-distributed random number of claims of risk $i(i=1, \ldots ., n)$. The $Y_{i}$ are assumed to be independent.

$$
\begin{gathered}
\boldsymbol{Y}=\left[\begin{array}{c}
Y_{1} \\
\cdot \\
\cdot \\
Y_{n}
\end{array}\right] \\
\lambda_{i}=e^{\sum_{j=1}^{r} x_{i j} b_{j}}
\end{gathered}
$$

$\lambda_{i}$ is the Poisson parameter of risk $i$. (2) shows that we assume the dependence of the expected number of claims on the tariff criteria to be multiplicative. The $x_{i j}$ are called covariates, the $b_{j}$ parameters. In the following we assume $x_{i 1}=1$ for all $i$. In this case the first parameter $b_{1}$ is called intercept.

$$
\boldsymbol{b}=\left[\begin{array}{c}
b_{1} \\
\cdot \\
\cdot \\
\cdot \\
b_{r}
\end{array}\right]
$$




$$
\boldsymbol{y}=\left[\begin{array}{l}
y_{1} \\
\cdot \\
\cdot \\
y_{n}
\end{array}\right]
$$

$f\left(y_{i}, \boldsymbol{b}\right)=e^{-\lambda_{i}} \frac{\lambda_{i}}{y_{i}} i \quad$ probability for risk $i$ to have $y_{i}$ claims

$L_{n}(\boldsymbol{b}, \boldsymbol{y})=\sum_{i=1}^{n} \ln \left(f\left(y_{i}, \boldsymbol{b}\right)\right) \quad$ log-likelihood function of $\boldsymbol{b}$

For $L_{n}(\boldsymbol{b}, \boldsymbol{y})$ to reach a maximum, the $r$ partial derivatives with respect to $b_{1}, \ldots, b_{r}$ must be equal to 0 . If we replace the observations $y_{i}$ by the random variables $Y_{i}$ in $L_{n}(\boldsymbol{b}, \boldsymbol{y})$, the partial derivatives are also random variables. Let $U_{n}(\boldsymbol{b})$ be the vector of the partial derivatives which is also called the score vector. Because the $Y_{i}$ are Poisson-distributed this vector is

$$
\boldsymbol{U}_{n}(\boldsymbol{b})=\left[\begin{array}{c}
\sum_{i=1}^{n} x_{i 1}\left(Y_{i}-\lambda_{i}\right) \\
\cdot \\
\cdot \\
\sum_{i=1}^{n} x_{i r}\left(Y_{i}-\lambda_{i}\right)
\end{array}\right] .
$$

If we require the partial derivatives of $L_{n}(\boldsymbol{b}, \boldsymbol{Y})$ to be equal to 0 , then the resulting $b_{1}, \ldots, b_{r}$ are also random variables which we designate as $B_{1}, \ldots, B_{r}$ and, when arranged in vector form, as $\boldsymbol{B}$.

Because the $Y_{i}$ are independent and because $\operatorname{Var}\left(Y_{i}\right)=\lambda_{i}$, the covariance of two elements of the score vector $\boldsymbol{U}_{n}(\boldsymbol{b})$, for instance the first and the second, is equal to

$$
E\left[\sum x_{i 1}\left(Y_{i}-\lambda_{i}\right) \sum x_{i 2}\left(Y_{i}-\lambda_{i}\right)\right]=\sum x_{i 1} x_{i 2} \lambda_{i}
$$

Let $\boldsymbol{Q}$ be the r-r-matrix with elements as in (3), i.e. $\boldsymbol{Q}=\operatorname{Cov}\left(\boldsymbol{U}_{n}(\boldsymbol{b})\right)$. In maximum likelihood theory, it is shown that the distribution of the vector $\boldsymbol{B}$, i.e. of the estimates of the parameters $b_{1}, \ldots, b_{r}$, is asymptotically normal (as $n \rightarrow \infty$ ), and that the inverse of $\boldsymbol{Q}$ tends to the covariance matrix of $\boldsymbol{B}$ :

$\boldsymbol{Q}^{-1} \rightarrow \operatorname{Cov}(\boldsymbol{B})$. 


\section{THE CASE OF CLASS VARIABLES WITH TWO LEVELS}

Following the example in the introduction the estimate for every frequency $\lambda_{i}$ should be close, e.g. within $c \lambda_{i}$ (e.g. $c=0.1$ ) to the true value with high probability (e.g. 95\%). For practical purposes we assume $\boldsymbol{B}$ actually follows a joint normal distribution with covariance matrix $\boldsymbol{Q}^{-1}$ although this holds true only asymptotically. In this case, according to (2), the logarithm of the frequency estimate of risk $i$ is the sum of $r$ normally distributed variables $x_{i j} B_{j}$ and, therefore, also normally distributed. The probability of the estimate of $\lambda_{i}$ to lie tolerably close to its expected value depends on the variance of $\sum x_{i j} B_{j}$. Writing $\boldsymbol{M}$ for $\boldsymbol{Q}^{-1}$ with elements $m_{j k}$ we have

$$
\operatorname{Var}\left(\sum_{j} x_{i j} \cdot B_{j}\right)=\sum_{j} \sum_{k} x_{i j} \cdot x_{i k} \cdot m_{j k}
$$

When the tariff criteria take on only two values, e.g. the driver's sex which is male or female, the place of residence (rural or urban), the car size (big or small), the engine size (large or small) and so on, then the covariates $x_{i j}$ have only two possible values for which it is convenient to choose 0 and 1 . Thus $x_{i j}=1$ when risk $i$ meets criterion $j$ and $x_{i j}=0$ otherwise. As can be seen from (3), in this case the elements $q_{j k}$ of $\boldsymbol{Q}$ represent the expected numbers of claims of risks which simultaneously meet criteria $j$ and $k$. The variance (4) is particularly simple if all $x_{i j}=1$ for $j=1, \ldots, r$. For such a risk (4) becomes

$$
\operatorname{Var}\left(\sum_{j} B_{j}\right)=\sum_{j} \sum_{k} m_{j k}
$$

There exists an upper bound for this variance since, as we are going to show in the following

$$
\sum_{j} \sum_{k} m_{j k} \leq \frac{1}{q_{11}}+\frac{1}{q_{22}}+\ldots \ldots+\frac{1}{q_{r r}} .
$$

Note that the $q_{j j}$ on the right side of the sign of inequality are the expected numbers of claims of risks which meet criterion $j$ and can be estimated with simple univariate statistics.

If in (4) some $x_{i j}=0$ then the variance of $\sum x_{i j} \cdot B_{j}$ cannot be estimated immediately by (5) and (6). We first have to replace the parameters $B_{j}$ by new parameters $B_{j}^{*}$ for which

$$
\sum_{j} x_{i j} \cdot B_{j}=\sum_{j} B_{j}^{*}
$$

before we can apply (6). The relation between $\boldsymbol{B}$ and $\boldsymbol{B}^{*}, \boldsymbol{B}=\boldsymbol{A} \cdot \boldsymbol{B}^{*}$, is given by an $\mathrm{r} \cdot \mathrm{r}$ matrix $\boldsymbol{A}$ which we define as follows: Let $s-1$ be the number of covariates which are equal to $0(2 \leq s \leq r)$, so that, possibly after renumbering the parameters $B_{j}$, 
$x_{i 1}=1$

$x_{i 2}=\ldots .=x_{i s}=0$

$x_{i, s+1}=\ldots .=x_{i r}=1$.

The elements of $\boldsymbol{A}$ are all equal to 0 except

$a_{j j}=1$ if $x_{i j}=1$

$a_{j j}=-1$ if $x_{i j}=0$

$a_{1 j}=1$ if $x_{i j}=0$.

Note that $\boldsymbol{A}=\boldsymbol{A}^{-1}$. Therefore, the solution $\boldsymbol{B}^{*}$ of $\boldsymbol{B}=\boldsymbol{A} \cdot \boldsymbol{B}^{*}$ can be easily calculated as

$$
\boldsymbol{B}^{*}=\left[\begin{array}{c}
B_{1}+B_{2}+\ldots+B_{s} \\
-B_{2} \\
-B_{3} \\
\cdot \\
\cdot \\
-B_{s} \\
B_{s+1} \\
\cdot \\
\cdot \\
B_{r}
\end{array}\right]
$$

Let $\boldsymbol{X}$ be the n $\cdot \mathrm{r}$ matrix of the covariates with respect to $\boldsymbol{B}$ and $\boldsymbol{Z}$ the $\mathrm{n} \cdot \mathrm{r}$ matrix of the covariates with respect to $\boldsymbol{B}^{*}$ defined by $\boldsymbol{Z}=\boldsymbol{X} \cdot \boldsymbol{A}$. As a result of the matrix multiplication $z_{i 1}=z_{i 2}=\ldots=z_{i r}=1$.

Let $Q^{*}$ be the matrix with elements $q_{j k}{ }^{*}$ defined as in (3) but with the covariates $z_{i j}$ instead of $x_{i j}$. Then $\boldsymbol{Q}^{*-1}$ is the covariance matrix of $\boldsymbol{B}^{*}$ and its elements $m_{j k}{ }^{*}$ fulfil (6) when $q_{11}, \ldots, q_{r r}$ are replaced by $q_{11}{ }^{*}, \ldots, q_{r r}{ }^{*}$ to the right of the inequality sign.

The transition from $\boldsymbol{X}$ to $\boldsymbol{Z}$ should be interpreted in the following way: If e.g. $x_{i 2}=1$ means risk $i$ is male, then $z_{i 2}=1$ means risk $i$ is female etc.

An upper bound (6) can be calculated for every risk $i$. The highest of these upper bounds is the one with the lowest $q_{j j}$. It is found in the following way:

$q_{11}$ is the total number of expected claims of the whole sample. $q_{i j}$, where $j>1$, is the number of expected claims of those risks which meet criterion $j$. The number of expected claims of risks which do not meet criterion $j$ is the difference $q_{11}-q_{j j}$. Define criterion $j$ so that $q_{j j} \leq q_{11}-q_{j j}$.

Before proving (6) let us look at a numerical example which is known to all readers who have learnt the theory of generalised linear models using SAS. In the Technical Report P-243 [2] the following example is given: 


\begin{tabular}{lclc}
\hline \hline risks & claims & car type & age group \\
\hline 500 & 42 & small & 1 \\
1200 & 37 & medium & 1 \\
100 & 1 & large & 1 \\
400 & 101 & small & 2 \\
500 & 73 & medium & 2 \\
300 & 14 & large & 2 \\
\hline \hline
\end{tabular}

By way of example let us look at the third segment, risks with large cars and age group 1, and estimate the variance of the logarithm of their frequency. In order to include an intercept term in the model we define $x_{i 1}=1$ for all $i$. Combining the car types small and medium into a new type "not large" we define $x_{i 2}=1$ if the car type is large and $x_{i 2}=0$ if it is not large; likewise $x_{i 3}=1$ if the age group is 1 and $x_{i 3}=0$ if it is 2 . Estimating the expected numbers of claims in $\boldsymbol{Q}$ by the observed numbers we get

$$
\boldsymbol{Q}=\left(\begin{array}{ccc}
\text { all } & \text { large } & \text { age 1 } \\
\text { large } & \text { large } & \text { large and age 1 } \\
\text { age 1 } & \text { large } \text { and age 1 } & \text { age 1 }
\end{array}\right)
$$

or, numerically instead of informally,

$$
\boldsymbol{Q}=\left(\begin{array}{ccc}
268 & 15 & 80 \\
15 & 15 & 1 \\
80 & 1 & 80
\end{array}\right)
$$

According to (6) the variance of the logarithm of the frequency estimate is at most equal to $1 / 268+1 / 15+1 / 80=0.08290$. A check with the covariance matrix in appendix 1 shows that a computer run does actually give a lower value for the estimated variance, namely 0.08217 .

In order to prove (6) we use some results from section 6 of chapter III (Normal Densities and Distributions) of the second volume of Feller [1]:

a) A symmetric, positive definite $r \cdot r$ matrix $\boldsymbol{Q}$ defines an $r$-dimensional normal density centred at the origin (Feller's theorem 4). The form of this density is $\varphi(x)=\gamma^{-1} e^{-\frac{1}{2} \boldsymbol{x}^{T} \boldsymbol{Q} x}$

where $\gamma$ is a constant and

$$
\boldsymbol{x}=\left[\begin{array}{c}
x_{1} \\
\cdot \\
\cdot \\
x_{r}
\end{array}\right]
$$


b) The vector $\boldsymbol{X}$ of the $r$ normally distributed random variables $X_{1}, \ldots, X_{r}$ has expectation $E(\boldsymbol{X})=\mathbf{0}$ and its covariance matrix $\boldsymbol{M}=\operatorname{Cov}(\boldsymbol{X})$ is the inverse of $\boldsymbol{Q}$ (Feller's theorem 3).

c) The variance of the marginal distribution of $X_{j}$ is $\operatorname{Var}\left(X_{j}\right)=1 / q_{j j}$ (Feller's theorem 1 and relation 6.5).

d) Expected value and variance of the conditional variable $X_{r} \mid X_{1}, \ldots, X_{r-1}$ are $E\left(X_{r} \mid X_{1}, \ldots, X_{r-1}\right)=-q_{1 r} / q_{r r} \cdot X_{1}-\ldots-q_{r-1, r} / q_{r r} \cdot X_{r-1}$ and

$\operatorname{Var}\left(X_{r} \mid X_{1}, \ldots, X_{r-1}\right)=1 / q_{r r}($ Feller's relation 6.13).

The definitions of $r, \boldsymbol{Q}$ and $\boldsymbol{M}$ used in this article are the same as in Feller, whereas the random variables $B_{j}-E\left(B_{j}\right)$ correspond to $X_{j}$ in Feller's notation. From (3) it is seen that our covariance matrix $\boldsymbol{Q}$ has the following properties:

$$
\begin{gathered}
q_{j j}>0 \text { for } j=1, \ldots, r \\
0 \leq q_{j k} \leq q_{j j}, q_{k k} \text { for } j \neq k .
\end{gathered}
$$

Moreover, $\boldsymbol{Q}$ is symmetric and positive definite. Therefore, according to a) it defines the density of an $r$-dimensional normal distribution.

Since Feller's variables $X_{j}$ are our $B_{j}-E\left(B_{j}\right)$ proving relation (6) is the same as proving

$$
\operatorname{Var}\left(X_{1}+X_{2}+\ldots+X_{r}\right) \leq 1 / q_{11}+1 / q_{22}+\ldots+1 / q_{r r} .
$$

We prove (9) by induction. For $r=1$ (9) reduces to c) and is true. Assume it is true for $r-1$. According to d) expected value and variance of the variable $X_{r} \mid X_{1}, \ldots, X_{r-1}$ are

$E\left(X_{r} \mid X_{1}, \ldots, X_{r-1}\right)=-q_{1 r} / q_{r r} \cdot X_{1}-\ldots \ldots \ldots-q_{r-1, r} / q_{r r} \cdot X_{r-1}$ and

$\operatorname{Var}\left(X_{r} \mid X_{1}, \ldots, X_{r-1}\right)=1 / q_{r r}$.

Therefore

$E\left(X_{1}+\ldots+X_{r} \mid X_{1}, \ldots, X_{r-1}\right)=\left(q_{r r}-q_{1 r}\right) / q_{r r} \cdot X_{1}+\ldots+\left(q_{r r}-q_{r-1, r}\right) / q_{r r} \cdot X_{r-1}$ and

$\operatorname{Var}\left(X_{1}+\ldots+X_{r} \mid X_{1}, \ldots, X_{r-1}\right)=1 / q_{r r}$.

Put for abbreviation $c_{j}=\left(q_{r r}-q_{j r}\right) / q_{r r}$.

Since for arbitrary conditional random variables $X \mid Y$ the relation

$\operatorname{Var}(X)=E[\operatorname{Var}(X \mid Y)]+\operatorname{Var}[\mathrm{E}(X \mid Y)]$ holds we have

$\operatorname{Var}\left(X_{1}+\ldots+X_{r}\right)=1 / q_{r r}+\operatorname{Var}\left(c_{1} \cdot X_{1}+\ldots+c_{r-1} \cdot X_{r-1}\right)$. We look for the coefficients $c_{j}$ which maximise this variance. Because of (7) and (8) we have $0 \leq c_{j} \leq 1$. Since for every $j(j=1, \ldots, r-1)$ the second derivative 


$$
\frac{\partial^{2}}{\partial c_{j}^{2}} \operatorname{Var}\left(c_{1} X_{1}+\ldots+c_{r-1} X_{r-1}\right)=2 \operatorname{Var}\left(X_{j}\right)>0
$$

the variance $\operatorname{Var}\left(c_{1} \cdot X_{1}+\ldots+c_{r-1} \cdot X_{r-1}\right)$ is maximal either for $c_{j}=0$ or $c_{j}=1$. If the $c_{j}$ are ordered appropriately then

$c_{1}=\ldots=c_{s}=1$, where $s \leq r-1$. The remaining $c_{j}=0$ (for $j>s$ ). Thus

$$
\begin{aligned}
\operatorname{Var}\left(X_{1}+\ldots+X r\right) & \leq 1 / q_{r r}+\operatorname{Var}\left(X_{1}+\ldots+X_{s}\right) \\
& \leq 1 / q_{r r}+1 / q_{11}+1 / q_{22}+\ldots+1 / q_{s s} \\
& \leq 1 / q_{r r}+1 / q_{11}+1 / q_{22}+\ldots+1 / q_{r-1, r-1}
\end{aligned}
$$

which proves (6).

\section{Class variables With MORE THAN TWO LeVels}

Class variables may assume more than two levels. For example the variable "car size" in [2] can have one of the three levels "small", "medium" or "large". A class variable $v$ with $k$ levels $(k>2)$ can be replaced by $k-1$ variables each having only 2 levels. In order to keep the notation simple, assume the covariates corresponding to these 2-level-variables are numbered $x_{i 2}, \ldots, x_{i k}$. This means that they immediately follow the covariate $x_{i 1}=1$ for the intercept term. Designate the $k$ levels by $l_{1}, \ldots, l_{k}$ and define the covariates $x_{i 2}, \ldots, x_{i k}$ as

$$
\begin{aligned}
x_{i 2}= & 1 \text { if } v=l_{1} \\
& 0 \text { otherwise }
\end{aligned}
$$

for $j=\underset{0}{3, \ldots, k x_{i j}=1}$ if $v \neq l_{j-1}$

Consequently, a risk $i$ for which the class variable $v$ is equal to $v=l_{1}$ has the covariates $x_{i 2}=\ldots=x_{i k}=1$.

In this way it is possible to apply the procedure of the previous section also to the case of general class variables. The contribution of the class variable $v$ to the upper bound (6) is $1 / q_{22}+\ldots+1 / q_{k k}$. From (3) and the definition of $x_{i 2}, \ldots, x_{i k}$ it can be seen that $q_{22}$ is the expected number of claims of risks with $v=l_{1}$. For $j>2, q_{j j}$ is the expected number of claims of risks with $v \neq$ $l_{j-1}$.

The last level, $l_{k}$, does not matter in the calculation of (6). If $l_{1}$ is given, we obtain the lowest value of (6) if we order the levels $l_{1}, \ldots, l_{k}$ so that $l_{k}$ is the level with the highest number of expected claims. In the numerical example, $l_{3}$ is thus the level "small" with 143 expected claims.

Ordering $l_{1}, \ldots, l_{k}$ so that $l_{1}$ is the level with the lowest number of expected claims leads to the highest upper bound (6). In fact: let $p_{2}$ be the expected number of claims in level $l_{1}, p_{3}$ the expected number of claims in level $l_{2}$ etc. Then the contribution of the class variable to (6) is 


$$
\frac{1}{p_{2}}+\frac{1}{q_{11}-p_{3}}+\ldots+\frac{1}{q_{11}-p_{k}} .
$$

Since $p_{2} \leq p_{j}$ for $j>2$, it follows that

$$
\frac{1}{p_{2}}+\frac{1}{q_{11}-p_{j}} \geq \frac{1}{p_{j}}+\frac{1}{q_{11}-p_{2}} .
$$

so that exchanging levels $l_{1}$ and $l_{j-1}$ would not increase the value of (6).

We illustrate the handling of class variables with more than two levels again using the numerical example from [2] and estimating expected numbers of claims by observed numbers.

The car size with the lowest number of expected claims is "large" and in age group 1 there are less expected claims than in age group 2. Therefore, the risk with the highest upper bound (6) is given by car size "large" and age group 1. We define the covariates

$$
\begin{aligned}
& x_{i 1}=1 \text { for the intercept term } \\
& x_{i 2}=1 \text { if car size }=\text { large } \\
& 0 \text { otherwise } \\
& x_{i 3}=1 \text { if car size not medium } \\
& 0 \text { otherwise } \\
& x_{i 4}=\begin{array}{l}
1 \text { if age group }=1 \\
0 \text { otherwise }
\end{array}
\end{aligned}
$$

Estimating the expected numbers of claims by inserting the observed numbers in the matrix $\boldsymbol{Q}$ we have

$$
\boldsymbol{Q}=\left(\begin{array}{cccc}
268 & 15 & 158 & 80 \\
15 & 15 & 15 & 1 \\
158 & 15 & 158 & 43 \\
80 & 1 & 43 & 80
\end{array}\right)
$$

According to (6) the variance of the logarithm of the frequency estimate is at most equal to $1 / 268+1 / 15+1 / 158+1 / 80=0.08923$ which is in fact higher than the value one obtains from the covariance matrix in appendix 2 , namely 0.08224 .

\section{AN UPPER BOUND FOR THE MINIMUM NUMBER OF OBSERVED CLAIMS NEEDED IN A SAMPLE}

We now return to the problem stated in the introduction: the estimate of $\lambda_{i}$ should lie with high probability $p$ (e.g. 95\%) close to its expected value (e.g. within $10 \%$ ). This means, writing $c$ for $10 \%$, the estimate $e^{B_{1}+B_{2}+\ldots+B_{r}}$ should not be lower than $(1-c) \cdot e^{b_{1}+b_{2}+\ldots+b_{r}}$ or higher than $(1+c) \cdot e^{b_{1}+b_{2}+\ldots+b_{r}}$. Consequently the exponent $B_{1}+B_{2}+\ldots+B_{r}$ which follows a normal distribution should not 
deviate from its expected value by more than $\ln (1-c)$. This defines the limit for the standard deviation of $B_{1}+B_{2}+\ldots+B_{r}$ : let $z_{p}$ be the value defined by $\operatorname{Prob}\left\{|Z| \leq z_{p}\right\}=p$, where $Z$ follows the standard normal distribution (in the example with $p=0.95$ and $c=0.1, z_{p}=1.96$ ).

We estimate the variance of $B_{1}+B_{2}+\ldots+B_{r}$ by the sum $u$ of the reciprocal diagonal elements of $\boldsymbol{Q}$. If $u \leq[\ln (1-c)]^{2} / z_{p}^{2}$ then our frequency estimate is sufficiently precise. Otherwise, assuming the composition of the sample remains the same, we determine a factor $f$ by which all diagonal elements of $Q$ are to be multiplied so that $u / f=[\ln (1-c)]^{2} / z_{p}^{2}$.

As a numerical example take again the motor insurance sample from [2] (see section 3). As has been shown in section 4 the segment of large cars and age group 1 has the highest upper bound of the variance, namely 0.08923 . The factor $f$ with which each $q_{j j}$ is to be multiplied in order to get the sufficiently large sample is

$f=z_{p}^{2} \cdot u /[\ln (1-c)]^{2}$

or in our numerical example $f=30.88$. The sample size needed is thus 30.88 times larger than the given sample with a total number of claims of $268 \cdot f=8,276$.

As one anonymous referee points out the upper bound of the variance is heavily influenced by $q_{22}=15$ corresponding to the segment of large vehicles. If we disregard the large vehicles and define $x_{i 2}$ to $x_{i 4}$ in another way so that e.g. for the car type with the second lowest number of claims (i.e. medium) and age group 1 all covariates are equal to 1 , then the total number of claims needed in the sample is much smaller $(2,715)$. In practice, we might do exactly that, i.e. tolerate that some rather insignificant tariff segments are less accurately rated provided that the accuracy of the important segments is sufficient.

\section{ACKNOWLEDGEMENT}

I would like to thank both anonymous referees for their constructive remarks that led to a better presentation of the paper.

\section{REFERENCES}

FelLeR, W. (1971) An Introduction to Probability Theory and Its Applications, volume 2, 2nd edition. Wiley.

SAS Technical Report P-243, SAS/STAT Software: The GENMOD Procedure, Release 6.09, Cary, NC: SAS Institute Inc., 1993, 88 pp.

HANS SCHMITTER

Swiss Reinsurance Company

Mythenquai 50/60 - CH-8022 Zurich

Tel. +4112854970

Telefax +4112824970

E-mail: Hans_Schmitter@swissre.com 


\section{APPENDIX 1}

data insure;

input $\mathrm{n}$ c car\$ age;

$\ln =\log (\mathrm{n})$

cards;

$\begin{array}{lrll}500 & 42 & \text { notlarge } & 1 \\ 1200 & 37 & \text { notlarge } & 1 \\ 100 & 1 & \text { large } & 1 \\ 400 & 101 & \text { notlarge } & 2 \\ 500 & 73 & \text { notlarge } & 2 \\ 300 & 14 & \text { large } & 2\end{array}$

The GENMOD Procedure

Model Information

Data Set

Distribution

Link Function

Dependent Variable

WORK.INSURE

Poisson

Offset Variable

$\log$

Observations Used

c

$\ln$

6

\section{Class Level Information}

\begin{tabular}{lrrr} 
Class & Levels & \multicolumn{2}{c}{ Values } \\
car & 2 & large & notlarge \\
age & 2 & 12 &
\end{tabular}

\section{Parameter Information}

$\begin{array}{llll}\text { Parameter } & \text { Effect } & \text { car } & \text { age } \\ \text { Prm1 } & \text { Intercept } & & \\ \text { Prm2 } & \text { car } & \text { large } & \\ \text { Prm3 } & \text { car } & \text { notlarge } & \\ \text { Prm4 } & \text { age } & & 1 \\ \text { Prm5 } & \text { age } & & 2\end{array}$

Estimated Covariance Matrix

$\begin{array}{lrrr} & \text { Prm1 } & \text { Prm2 } & \text { Prm4 } \\ \text { Prm1 } & 0.005710 & -0.005293 & -0.005637 \\ \text { Prm2 } & -0.005293 & 0.07164 & 0.004298 \\ \text { Prm4 } & -0.005637 & 0.004298 & 0.01808\end{array}$




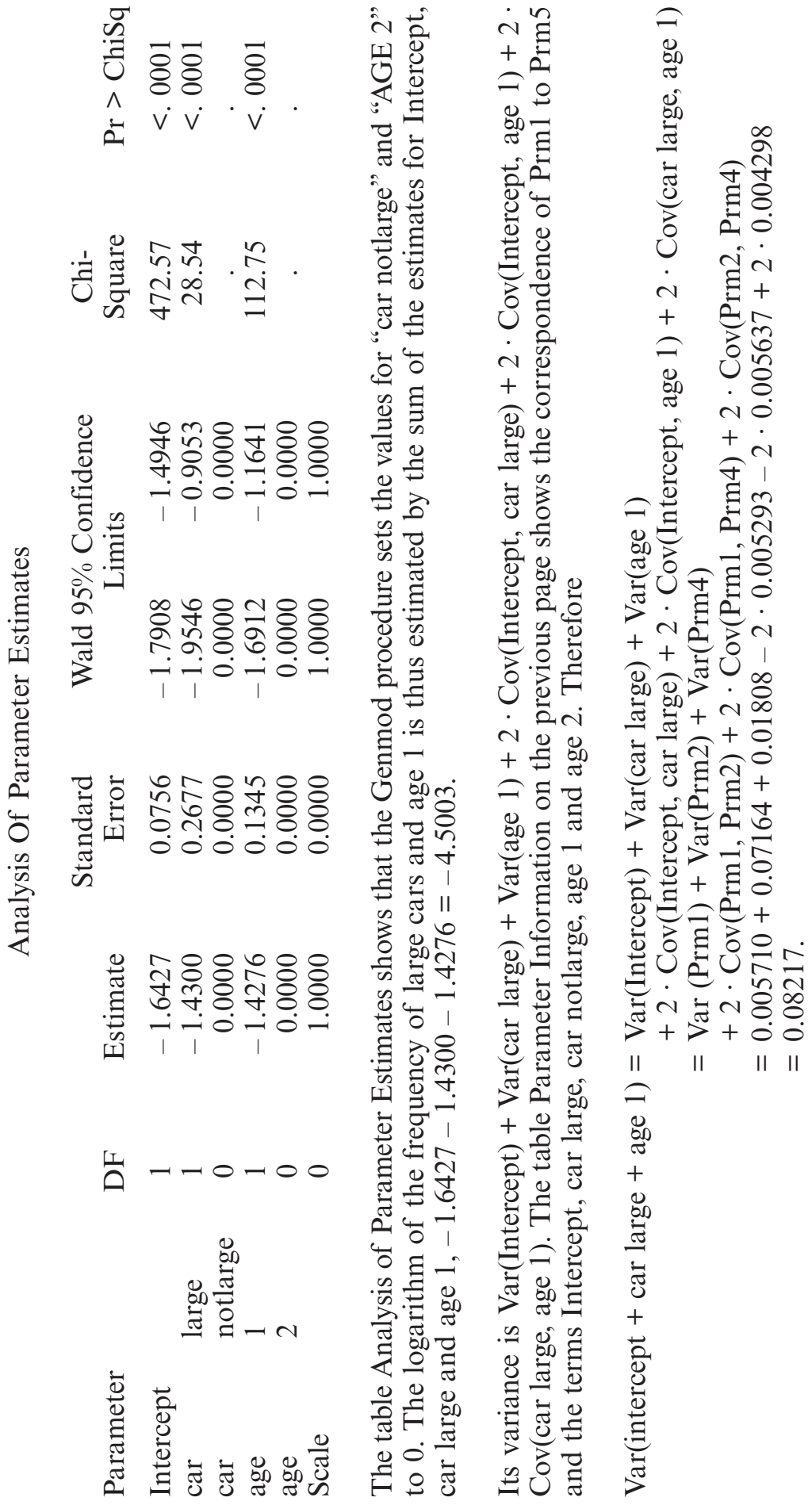




\section{APpendix 2}

data insure;

input $\mathrm{n}$ c car\$ age;

$\ln =\log (\mathrm{n})$

cards;

$\begin{array}{lrll}500 & 42 & \text { notlarge } & 1 \\ 1200 & 37 & \text { notlarge } & 1 \\ 100 & 1 & \text { large } & 1 \\ 400 & 101 & \text { notlarge } & 2 \\ 500 & 73 & \text { notlarge } & 2 \\ 300 & 14 & \text { large } & 2\end{array}$

The GENMOD Procedure

Model Information

Data Set

Distribution

Link Function

Dependent Variable

Offset Variable

Observations Used
WORK.INSURE

Poisson

Log

$\ln$

6

Class Level Information

$\begin{array}{lrl}\text { Class } & \text { Levels } & \text { Values } \\ \text { car } & 3 & \text { large medium small } \\ \text { age } & 2 & 12\end{array}$

Parameter Information

$\begin{array}{llll}\text { Parameter } & \text { Effect } & \text { car } & \text { age } \\ \text { Prm1 } & \text { Intercept } & & \\ \text { Prm2 } & \text { car } & \text { large } & \\ \text { Prm3 } & \text { car } & \text { medium } & \\ \text { Prm4 } & \text { car } & \text { small } & \\ \text { Prm5 } & \text { age } & & 1 \\ \text { Prm6 } & \text { age } & & 2\end{array}$

Estimated Covariance Matrix

$\begin{array}{lrrrr} & \text { Prm1 } & \text { Prm2 } & \text { Prm3 } & \text { Prm5 } \\ \text { Prm1 } & 0.008150 & -0.007772 & -0.006344 & -0.004623 \\ \text { Prm2 } & -0.007772 & 0.07418 & 0.006556 & 0.003113 \\ \text { Prm3 } & -0.006344 & 0.006556 & 0.01645 & -0.002592 \\ \text { Prm5 } & -0.004623 & 0.003113 & -0.002592 & 0.01847\end{array}$




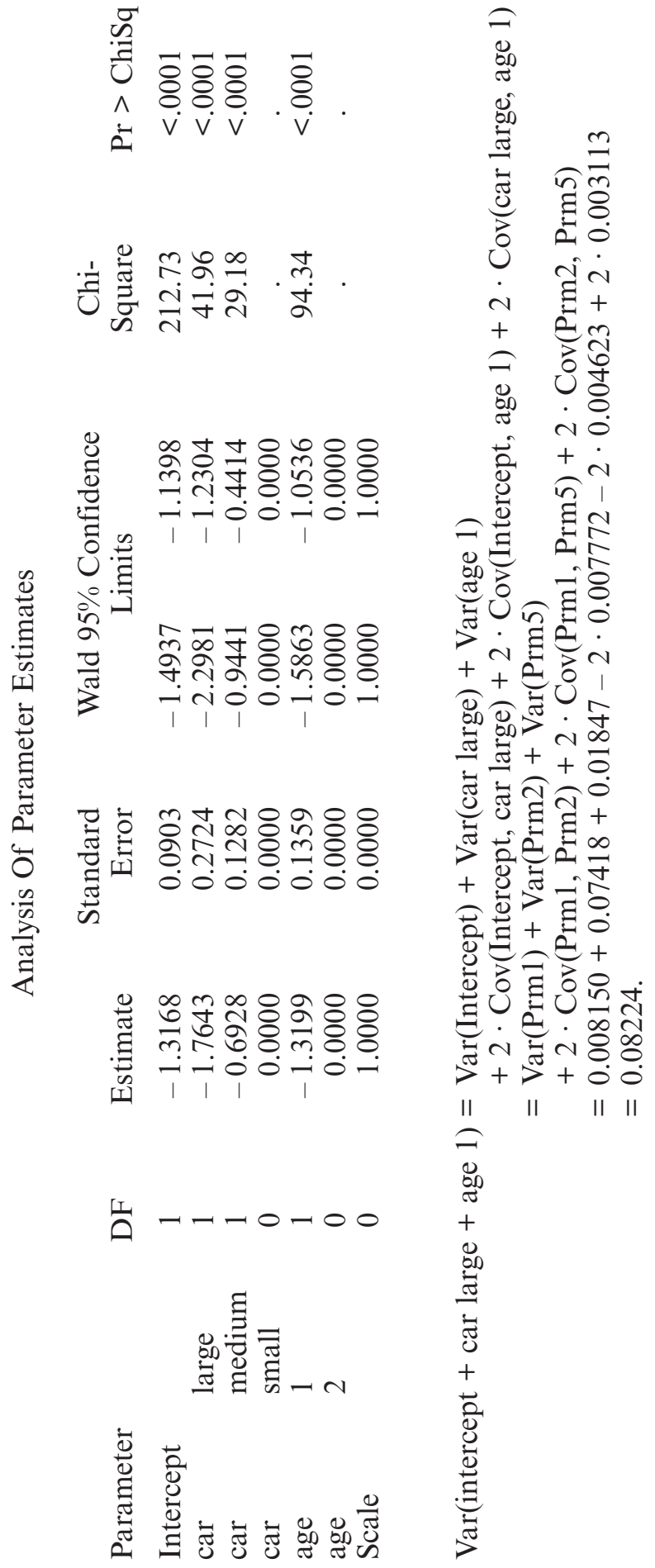

